\title{
Liver disease in infancy: a 20 year perspective
}

\author{
Giorgina Mieli-Vergani, Edward R Howard, Alex P Mowat
}

In Northern Europe and North America the majority of children with chronic or life threatening liver disease first develop features of liver disease in early infancy. They usually have a hepatitis syndrome characterised by conjugated hyperbilirubinaemia, abnormal biochemical tests of liver function, hepatomegaly with or without splenomegaly and partial or complete cholestasis. There may be features caused by malabsorption, particularly of fat soluble vitamin $\mathrm{K}$. It is from this cohort that the majority of children requiring transplantation are drawn. Such liver disease may occur in as many as 1 in 250 newborn infants. ${ }^{12}$

During the development of the paediatric liver service at King's, in fruitful cooperation with the adult Liver Unit, we have identified important differences in some aspects of the clinical and pathobiological features of chronic liver disease which occur in children and adults, such as autoimmune chronic active hepatitis and primary sclerosing cholangitis. ${ }^{34}$ Our major efforts, however, have been to identify and improve the treatment of those with progressive liver disease starting in infancy. These account for over $50 \%$ of patients referred, the numbers in the main categories being given in the Table. This review provides an opportunity to consider some developments in which we have participated in the last 20 years.

We highlight four advances which have had a major impact on the management of these disorders. Most important has been the observation that a large proportion of patients with biliary atresia could have prolonged survival with a good quality of life after successful porto-enterostomy. The second has been identifying the role of alpha-1-antitrypsin deficiency (PIZZ) as a genetic factor associated with a particularly severe form of the syndrome frequently leading to cirrhosis. The third has been the developments in molecular biology which have led to the identification of rare genetic or congenital disorders presenting as neonatal hepatitis, gradually reducing the percentage of benign cryptogenic cases. Three will be considered: Niemann-Pick type 2, Zellweger's syndrome and Alagille's syndrome. Lastly, there has been the increased awareness of the importance of liver disease in children and the provision of improved treatment and research facilities which has come from the activities of the Children's Liver Disease Foundation.

Health and Sur Cry King's College School of Medicine and Dentistry, London SE5 9RS Giorgina Mieli-Vergani E R Howard Alex P Mowat Correspondence to: Alex P Mowat Department of Child Health, Variety Club Children's Hospital, King's College Hospital, Denmark Hill, London SE5 9RS
In the last 30 years it has been confirmed that the underlying pathological process in this disorder process. It initially causes atresia of all or part of the extrahepatic biliary system which in uncorrected cases extends into the major intrahepatic is usually a destructive sclerosing inflammatory ducts. A biliary cirrhosis rapidly develops with a mean age of death of 11 months with less than $5 \%$ surviving beyond two years. ${ }^{5} \mathrm{Up}$ to $25 \%$ of patients have congenital abnormalities in cardiovascular, gastrointestinal and genitourinary systems with up to $7 \%$ having a distinct constellation of abnormalities forming the polysplenia syndrome which may include situs inversus abdominis, preduodenal portal vein and intestinal malrotation as well as multiple or absent spleen. ${ }^{6}$ The aetiology of biliary atresia remains unknown. Those with other abnormalities may be a separate aetiological subgroup.

The vast majority of these infants are entirely well during the first four to eight weeks of life apart from their jaundice. Their wellbeing often causes paediatricians and other health workers to dismiss consideration of this disorder until successful surgery is less likely, the process having destroyed the major intrahepatic bile ducts. Kasai and his coworkers in the late 1950's pioneered the operation of portoenterostomy. ${ }^{8}$ In this procedure an anastomosis is fashioned between the area of the porta hepatis from which the inflamed bile duct remnants have been resected and a $30-40 \mathrm{~cm}$ Roux loop. This allows bile to drain from patent major intrahepatic bile ducts directly into the bowel. Before Kasai's work less than $15 \%$ of patients with biliary atresia were operated on at the stage in the development of the process in which the surgeon could identify a bile-containing duct at the porta hepatis and the surgeon was able to fashion a direct bile duct to bowel anastomosis. With this procedure a few patients cleared their jaundice and achieved longterm survival.

The portoenterostomy procedure was introduced only slowly into the surgical practice of Europe and North America. ${ }^{5}$ The first 32 patients seen between 1970 and 1973 in the newly established children's liver service at King's College Hospital all died of liver failure. ${ }^{9}$ Surgical experience with an experimental model of this condition in the pig, however, led to improvements in technique and the first longterm survivor (Fig 1) was treated in $1973 .{ }^{5}$ Since then it has been possible to obtain good bile flow with normal serum bilirubin values within one to six months in between $80 \%$ and $90 \%$ of infants if they are operated on by 60 days of age. In infants operated on later the percentage becoming jaundice free is between $20 \%$ and $35 \%$. Results are less satisfactory in centres with less experience. ${ }^{10}$

Biochemical tests of liver function remain abnormal for many years after surgery. Hepatic fibrosis is usually well established at the time of surgery and is aggravated if ascending cholangitis occurs. Nevertheless in infants becoming jaundice free the 15 years survival with a good quality of life is now almost $90 \% .{ }^{11}$ In infants in whom the bilirubin is not reduced the rate of progression to cirrhosis is not slowed. If bile drainage is 


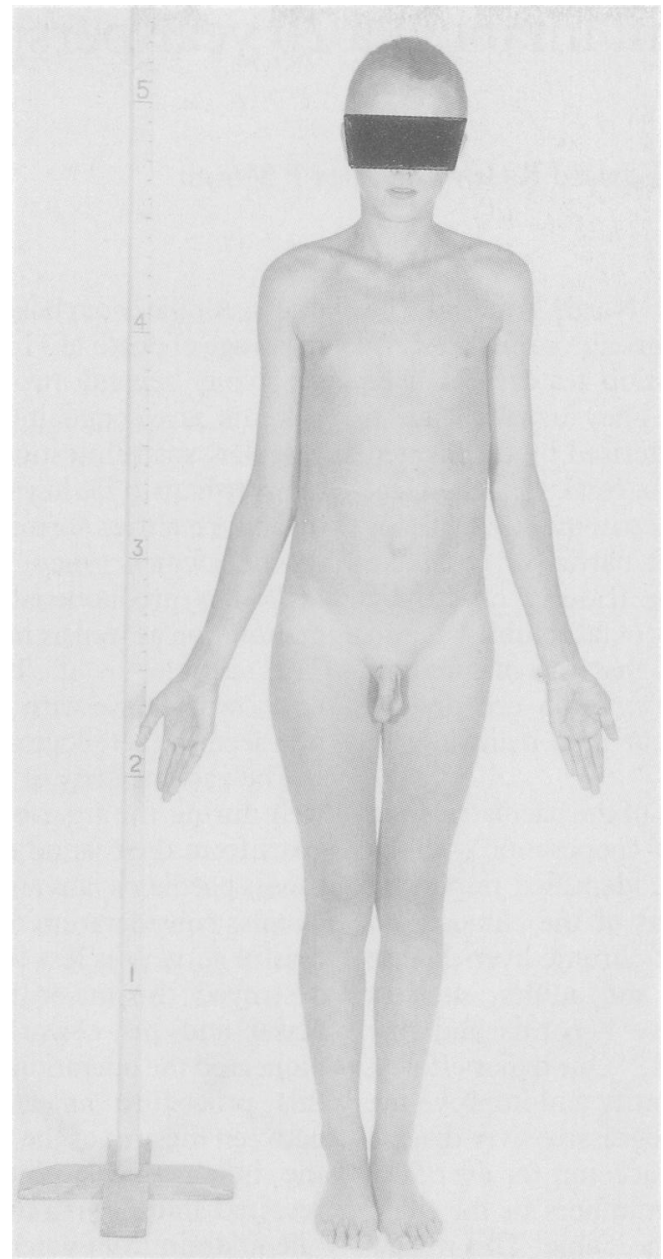

Figure 1: Fifteen year old boy with extra hepatic biliary atresia corrected at the age of 10 weeks.

partially effective death may be delayed up to the second decade. ${ }^{11}$

Cholangitis is a major and unresolved problem after portoenterostomy. It occurs in over $50 \%$ of cases in the first two years after surgery. It is characterised by fever, recurrence or aggravation of jaundice and frequently features of septicaemia. Blood culture, ascitic aspirate or liver biopsy may identify the organism responsible. Broad spectrum antibiotic therapy is given pending in vitro sensitivity results. Neither prophylactic antibiotics nor complicated surgical techniques of cutaneous diversion of bile influence the frequency or severity of cholangitis. ${ }^{12}$

With successful surgery portal pressure falls, but approximately $50 \%$ of those aged five years have oesophageal varices. Ten per cent to $15 \%$ have alimentary bleeding. We have shown that injection sclerotherapy is a very satisfactory method of management of this complication and devascularisation or shunt procedures are rarely needed. ${ }^{13}$

All require dietary supplements because of malabsorption in the first year of life. Thereafter normal growth can be expected in these patients even though there may be severe intrahepatic fibrosis or even cirrhosis.

In approximately $10 \%$ of cases in whom the serum bilirubin returns to normal cirrhosis progresses either because of the ascending cholangitis or possibly the continuation of the original cholangiopathy. For these patients and for those in whom surgery has not been effective in returning the serum bilirubin to normal liver transplantation is now possible. The morbidity and mortality of portoenterostomy (and the cost) is minor to that of transplantation, making portoenterostomy the initial procedure of choice in all except infants who have decompensated cirrhosis when they first present. ${ }^{14}$ is Transplantation should be considered for the infant in whom portoenterostomy fails completely or for the longterm survivor with deteriorating liver function or life threatening portal hypertension. Sadly, biliary atresia remains the most frequent reason for transplantation in children in the United Kingdom because of delay in initial recognition of the possibility of serious hepatobiliary disease in infants remaining jaundiced after two weeks of age.?

\section{Alpha-1-antitrypsin deficiency and liver disease}

Alpha-1-antitrypsin was isolated by Schultz and coworkers in $1955 .{ }^{16}$ An association with liver disease and alpha-1-antitrypsin deficiency was first identified in two brothers with cirrhosis. ${ }^{17}$ Since then genetic deficiency of alpha-1antitrypsin has had a major impact in the clinical practice of paediatric hepatology. ${ }^{18-20}$ The deficiency state PIZZ (PI=protease inhibitor, phenotype $\mathrm{ZZ}$ ) which is inherited in an autosomal fashion is the second most common single diagnosis after biliary atresia in infants with the hepatitis syndrome in populations of European descent. Alpha-1-antitrypsin phenotyping, preferably by isoelectric focusing, is required for diagnosis. Serum concentrations, measured by immunological techniques, may be increased or decreased by associated diseases or drugs and therefore unreliable in making a diagnosis. ${ }^{18}$

The deficient infant has an increased susceptibility to liver disease with $15 \%$ developing clinical features of a hepatitis. ${ }^{21} 22$ What initiates the liver damage is controversial..$^{20}$ Alpha-1antitrypsin is thought to inhibit tissue-damaging proteases. It inhibits a wide range of serine proteases, particularly neutrophil elastase. This enzyme functions as an extracellular protease. Its prime substrate is elastin but it also attacks many other proteins including a variety of proteins in the coagulation and complement cascades, $E$ coli cell wall components and all major components of extracellular matrix. ${ }^{1824}$ In the deficient individual uninhibited action of proteases may cause progressive liver disease. The HLA status may contribute to the severity of liver injury. ${ }^{25}$

Liver disease is most commonly identified in early infancy as a conjugated hyperbilirubinaemia with hepatitis in $11 \%$ or a bleeding state caused by vitamin $\mathrm{K}$ malabsorption in $2 \%$. Up to $70 \%$ have abnormal biochemical tests of liver function in early infancy. The mean age at recognition of icteric hepatitis is between two and three weeks. Jaundice lasts on average for three months but may persist for as long as a year. The infants commonly have slow weight gain, some may show irritability or lethargy. They are at risk of septicaemia which can cause a devastating deterioration in liver function with marked pro- 


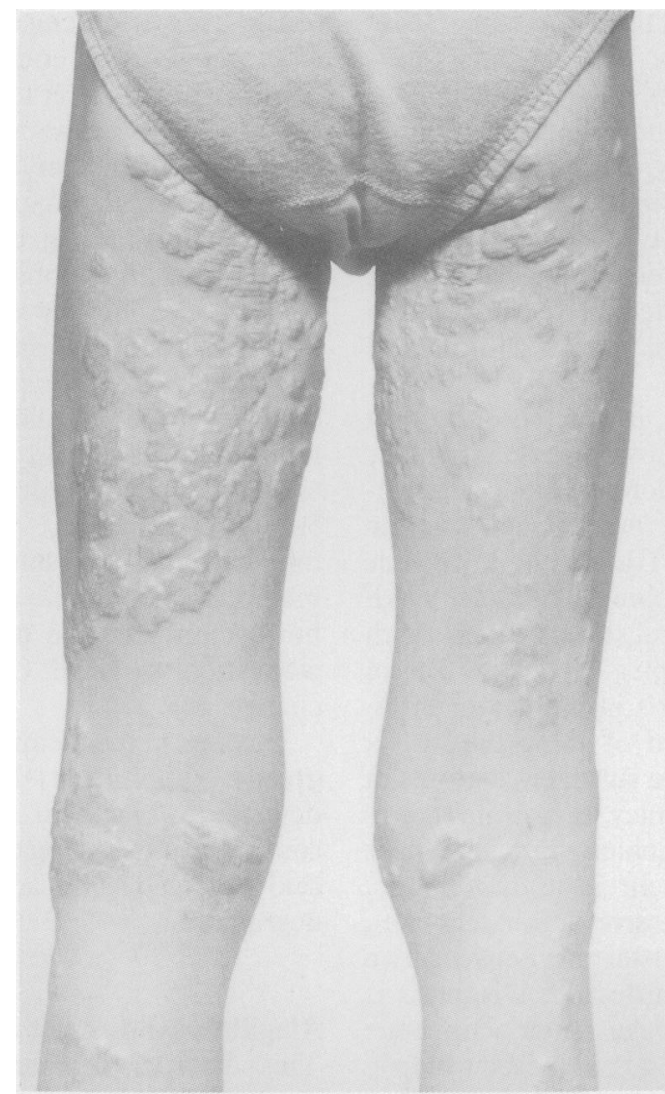

Figure 2: Xanthelasma in a 10 year old boy with Alagille's syndrome.

longation of the prothrombin time. All have hepatomegaly and approximately $50 \%$ have splenomegaly. Those presenting with a severe bleeding episode do so at two to six weeks of age. The prothrombin time (prothrombin ratio) is greatly prolonged. It reverts to normal within six hours with intravenous vitamin $\mathrm{K}$. Rarely the presentation is with ascites in the newborn period. The course of the liver disease is independent of the mode of presentation in infancy.

About 5\% remain jaundiced, progress to decompensated cirrhosis and die in the first year of life. The remainder recover from the acute hepatitis and in approximately $25 \%$ the clinical and biochemical abnormalities gradually improve and results come within the normal range at ages ranging from three to 10 years. Survival into the third decade without features of cirrhosis has been recorded in such patients.

Approximately $25 \%$ have died from complications of cirrhosis at ages ranging from six months to 17 years. Death from liver disease occurs within two months to four years of the onset of complications. Haematuria and/or albuminuria as a result of glomerular lesions is a late complication which may predispose to severe systemic hypertension after transplantation. ${ }^{28}$

TABLE Infants with conjugated hyperbilirubinaemia, referred to King's College Hospital between 1970-90

Biliary atresia

Idiopathic hepatitis of infancy

Alpha-1-Antitrypsin deficiency

Other hepatitis of infancy

Alagille's syndrome

Choledochal cyst
Approximately $25 \%$ survive through the first decade although they have histologically confirmed cirrhosis. A further $25 \%$ without liver biopsy evidence of cirrhosis have persistently abnormal liver function tests with or without clinical features of portal hypertension. In some of these without clinical abnormality, liver function test may eventually become normal but the outcome for those with clinical or biochemical evidence of liver disease is guarded. ${ }^{18} 1926-28$

The management is that of chronic cholestasis and of cirrhosis. ${ }^{2}$ There is no specific treatment for liver disease associated with alpha-1antitrypsin deficiency short of liver transplantation. It corrects the serum phenotype to that of the donor. ${ }^{29}$ The longest follow up is only 16 years making it too early to determine whether it will prevent emphysema. ${ }^{30}$ In up to $80 \%$ of children within a sibship the liver disease is of the same severity as the proband.$^{26}$ Antenatal diagnosis of the PIZZ state initially possible only with fetal blood sampling ${ }^{31}$ is now possible by examining the DNA of chorionic villus samples using synthetic oligonucleotide probes specific for the $\mathrm{M}$ and $\mathrm{Z}$ gene or by restriction fragment length polymorphism. With the polymerase chain reaction results can be made available within a few days of sampling at 11 weeks gestation. Preliminary studies confirm the validity of such techniques. ${ }^{32}$

In adults with emphysema it has been possible to bring plasma alpha-1-antitrypsin levels up to normal values with infusions of plasma derived alpha-1-antitrypsin. This has not been shown to modify the emphysema. Nor did it decrease to normal levels the raised serum transaminases values found in a few patients who were treated in this fashion. ${ }^{33}$

Direct gene targeting to the liver in vitro is another possibility having been used successfully to stimulate albumin production in analbuminaemic rats. ${ }^{34}$ The gene was contained in a plasmid which is targeted to the hepatocytespecific asialoglycoprotein receptor and carried into the hepatocyte by pinocytosis. In this way the PIMZ state would be created. Another theoretical possibility is the correction of the hepatic secretory problem by insertion of another gene which makes a complementary change in the polypeptide allowing it to assume normal tertiary configuration which may facilitate secretion. ${ }^{33} 35$ Perhaps more immediately practicable and attractive, to paediatric hepatologist if not to pulmonary physicians, would be a trial of exogenous serum-derived alpha-1-antitrypsin, given iv at one to four weekly intervals, as is being used in emphysema but commencing as soon as significant liver damage is identified, perhaps in conjunction with a neonatal screening programme. It should be possible to conduct a trial of therapy lasting six to 12 months which in the course of at most a decade, and perhaps much shorter, would indicate whether such therapy was efficacious.

\section{Niemann-Pick type 2}

The Niemann-Pick group of diseases are sphingomyelin-cholesterol lipidosis. ${ }^{36}$ Failure of lipid degradation causes abnormal lipid storage 
with hepatosplenomegaly, the formation of foam cells in the bone marrow and in some forms progressive central nervous system involvement. In type 1 disease sphingomyelinase activity is $<10 \%$ of normal. In type 2 disease sphingomyelinase activity is normal except in cultured fibroblasts in which activity may be reduced by $80 \%$. A defect in cholesterol esterification has recently been demonstrated in vitro. ${ }^{37}$ Within both types there is considerable clinical and biochemical variability.

Niemann-Pick type 2 is the second most common genetic cause of liver disease in infancy in the United Kingdom, after alpha-1antitrypsin deficiency. It has previously been designated type C, D, III, IV and juvenile Niemann-Pick with sea blue histiocytes, DAF syndrome and neurovisceral storage disease with supranuclear ophthalmoplegia. Over $50 \%$ of cases present with hepatitis in infancy or intrauterine ascites. ${ }^{3738} \mathrm{~A}$ third of these die by six months of age while in the survivors features of liver disease regress in infancy leaving an asymptomatic cirrhosis. Unfortunately they, like those in whom liver involvement is asymptomatic, develop features of progressive, ultimately fatal, neurological involvement starting between two years of age and the sixth decade. Diagnosis is suspected by finding storage material in bone marrow and within neurones in a rectal biopsy and is confirmed by observing defective cholesterol esterification in cultured fibroblasts. There is no treatment. Liver transplantation does not arrest the disease. Diagnosis is essential for genetic counselling. Prenatal diagnosis by amniocentesis is possible.

\section{Zellweger's syndrome}

Zellweger's syndrome, an autosomal recessive disorder, is characterised by absence of peroxisomes and defective function of peroxisomal enzymes. ${ }^{3940}$ It is a multisystem disorder with profound abnormalities of neuronal migration and brain structure, hepatic malfunction, renal cortical cysts, abnormal calcification, retinal degeneration and multiple congenital anomalies. The defect is associated with a sometimes diagnostic facial appearance, severe mental retardation, multiple congenital anomalies, including renal cortical cysts, retinal degeneration and deafness. ${ }^{37}{ }^{38} \mathrm{It}$ is the most severe of the disorders of peroxisome biosynthesis. Hepatomegaly and raised transaminases with jaundice in approxmately $60 \%$ of cases are the initial hepatic features. In the first weeks of life there is mild periportal fibrosis with an irregular relationship between portal tracts and central veins but by eight weeks of age fibrosis is more marked and frequently a micronodular cirrhosis is established by 20 weeks of age. Eighty per cent of patients die by six months of age, with few surviving beyond two years, but less severe incomplete forms of the condition, with more prolonged survival are increasingly described. There is no treatment for this condition but prenatal diagnosis is possible. The main scientific interest in the condition has been to identify the key metabolic role of peroxisomes. They account for an estimated $20 \%$ of the oxygen consumption of the liver being engaged in the oxidation of a large number of endogenous and exogenous substrates. The latter include ethanol, methanol, and nitrites. Peroxisomes also have an important role in the catabolism, by beta oxidation, of fatty acids of chain length greater than 12 (producing much energy), long chain dicarboxylic acids, prostaglandins, xenobiotics and the side chain of cholesterol. They have an essential role in the synthesis of the plasmalogens (phospholipids) and bile acids. In this syndrome beta oxidative cleavage of the side chain does not occur. Therefore $5 \beta$-cholestane- $3 \alpha, 7 \alpha, 12 \alpha$-triol is oxidised to $3 \alpha, 7 \alpha, 12 \alpha$-trihydroxy-5ß-cholestanoic acid, $5 \alpha$ cholestane- $3 \alpha, 7 \alpha$-diol to $3 \alpha, 7 \alpha$ dihydroxy-5ß-cholestanoic acid. These are not metabolised to cholic acid or desoxycholic acid but accumulate and undergo either side chain elongation to form $\mathrm{C}_{29}$ bile acids or nuclear changes.

Diagnostic biochemical abnormalities include greatly diminished levels of plasmalogens, defects in bile acid synthesis, raised serum concentrations of long chain fatty acids, phytanic acid and pipecolic acid (a product of lysine degradation).

\section{Alagille's syndrome}

This disorder is characterised by paucity of interlobular bile ducts (intrahepatic bile duct hypoplasia) occurring in association with a range of characteristic cardiovascular, skeletal and ocular anomalies (arteriohepatic dysplasia, syndromic paucity of the interlobular bile ducts). ${ }^{41}{ }^{42}$ The syndrome is probably inherited in an autosomal dominant fashion with variable expression. The family history is positive in $15 \%$ of cases, although full expression of the syndrome may not be present in earlier generations.

Essential to the diagnosis is a decrease in the number of interlobular bile ducts seen in portal tracts (ratio $<0.06$ ). Such paucity may be found also in association with genetic disorders such as alpha-1-antitrypsin deficiency or failure of primary bile salt synthesis, in chromosomal abnormalities and rarely with intrauterine infections. It is frequently present in biliary atresia after nine months of age. Diagnosis, based on histological finding, is supported by the finding of the typical facies, deep set eyes, mild hypertelorism, overhanging forehead, a straight nose which in profile is in the same plane as the forehead and a small pointed chin, posterior embryotoxon, vertebral body defects on spinal radiographs and the presence of a cardiac murmur. Peripheral pulmonary stenosis is the most common abnormality but a wide range of other forms of cyanotic and acyanotic heart disease may occur. As none of these abnormalities are unique to this syndrome if the biopsy findings are not typical (see below) and cholestasis is complete ERCP may be required to distinguish this disorder from biliary atresia. $^{43}$

There is long standing cholestasis varying in severity from mild pruritus to life long jaundice. Severe cases start with jaundice in the neonatal period, developing pruritus at five months by which time there is hypercholesterolaemia (frequently $>15 \mathrm{mmol} / \mathrm{l}$ ) with xanthelasma appear- 
ing six to 12 months later. Xanthelasma usually clear some years before the jaundice. Less severe cases have no xanthelasma. Jaundice may persist, but in others clears in late childhood or early adult life. Renal impairment and renal tubular acidosis may occur in association with 'mesangiolipidosis' or juvenile nephronophthisis. The longterm prognosis is uncertain but some $15 \%$ may go on to develop cirrhosis and $5 \%-10 \%$ die from liver disease. In one series $25 \%$ died from cardiac involvement, classically a peripheral pulmonary stenosis, or infection. ${ }^{41}$ The pathogenesis is intriguing in that during the first three to nine months of age it may be impossible to make the histological diagnosis in up to $25 \%$ of cases because bile ductular proliferation with features suggestive of bile duct obstruction may be the main finding. If the jaundice clears all features of liver disease may eventually remit and where liver biopsies have been performed the bile duct to portal tract ratio has been normal.

The treatment is that of chronic cholestasis with particular emphasis on adequacy of fat soluble vitamin replacement, particularly vitamin E. After vitamin E deficiency for periods ranging from six months to four years these patients develop a neuromuscular degenerative syndrome. This can be prevented if the serum vitamin $\mathrm{E}$ concentration is maintained within the normal range. Growth failure and pruritus are major problems. It is not clear whether growth failure can be reversed by liver transplantation.

\section{The Children's Liver Disease Foundation}

In the last decade paediatric hepatology in the United Kingdom has received a considerable boost from the activities of the Children's Liver Disease Foundation. This organisation has as its aims the improved management of liver disease in children by provision of better facilities, promotion of research and teaching and by giving emotional support for the families of children affected. Parents and friends of children with liver disease have been largely responsible for collecting the magnificent sum of $£ 1.2$ million pounds which has been spent in furthering these aims. To them and to the trustees we owe a large debt of gratitude. At last there is increasing professional and public awareness of the problem of liver disease in children. We hope that the British Liver Foundation can achieve as much for adult hepatology.

1 Fitzgerald JF. Cholestatic disorders in infancy. Ped Clin N Am 1988; 35: 357-73.

2 Mowat AP. Liver disorders in Childhood. London: Butterworths, 1987

3 El Shabrawi M, Wilkinson M, Portmann B, et al. Primary sclerosing cholangitis in Childhood. Gastroenterology 1987; 92: 1226-35.

4 Mieli-Vergani G, Lobo-Yeo A, McFarlane BM, McFarlane IG, Mowat AP, Vergani D. Different immune mechanisms leading to autoimmunity in primary sclerosing cholangitis and autoimmune chronic active hepatitis of childhood. Hepatology 1989; 9: 198-203.

5 Howard ER. Biliary atresia. In: Blumgart LH, ed. Surgery of the liver and biliary tract. Edinburgh: Churchill Livingstone, 1988: 707-22.

6 Chandra RS. Biliary atresia and other structural anomalies in the congenital polysplenia syndrome. $f$ Pediatr 1974; 85: 649-55.

7 Mieli-Vergani G, Howard ER, Portmann B, Mowat AP. Late referral for biliary atresia - missed opportunities for effective surgery. Lancet 1989; i: 421-3.
8 Kasai M, Suzuki S. A new operation for 'non-correctable' biliary atresia. Shujitsu 1959; 13: 173-9.

9 Mowat AP, Psacharopoulos HT, Williams R. Extrahepatic biliary atresia versus neonatal hepatitis. A review of 137 prospectively investigated infants. Arch Dis Child 1976; 51: 763-70.

10 McClement J, Howard ER, Mowat AP. Results of surgical treatment for extrahepatic biliary atresia in United Kingdom 1980-82. BMF 1985; 290: 345-7.

11 Ohkohchi N, Chiba T, Ohi R, Mori S. Long-term follow-up study of patients with cholangitis after successful Kasai operation in biliary atresia: selection of recipients for liver transplantation. $\mathcal{F}$ Pediatr Gastroenterol Nutr 1989; 9: 41620.

12 Ecoffey C, Rothman E, Bernard O, Hadchouel M, Valayer J, Alagille D. Bacterial cholangitis after surgery for biliary atresia. F Pediatr 1987; 111: 824-9.

13 Stringer MD, Howard ER, Mowat AP. Endoscopic sclerotherapy in the management of esophageal varices in 61 children with biliary atresia. F Pediatr Surg 1989; 24: 438-42.

14 Anonymous. Long term survival in biliary Atresia. Lance 1989; ii: $597-8$.

15 Alagille D, Laurent J, Roy CC. Is there still a place for the Kasai procedure in the treatment of biliary atresia? $\mathcal{F}$ Pediatr Gastroenterology 1989; 9: 405-6.

16 Schultze HE, Gollner I, Heide K, Schonberger M, Schwick G. Zur Kenntis der alpha globuline des menschlichen normalserums. Z Naturforsch 1955; 10: 463-9.

17 Freier E, Sharp HL, Bridges RA. Alpha-1-antitrypsin deficiency associated with familial infantile liver disease. Clin ciency associated with

18 Cox DW. Alpha-1-antitrypsin deficiency. In: Scriver CR, Beautt AL, Sly WS, Valle D, eds. The metabolic basis of inherited disease. 6th ed. New York: McGraw-Hill, 1989: 2409-37.

9 Mowat AP. Alpha-1-antitrypsin deficiency in liver disease. In Williams R, Madre WC, eds. Gastroenterology. Vol 4, 1 (Butterworths International Medical Reviews) London: Butterworths, 1984: 52-75.

20 Hussain M, Mieli-Vergani G, Mowat AP. Alpha-1-antitrypsin deficiency and liver disease. Clinical presentation, diagnosis and treatment. $\mathcal{F}$ Inher Metab Dis (in press).

21 Sveger T. The natural history of liver disease in alpha-1antitrypsin deficient children. Acta Paediatr Scand 1988; 77 . 847-51.

22 Psacharopoulos HT, Mowat AP. Incidence and early history of obstructive jaundice in infancy in SE England. In: Neonatal hepatitis and biliary atresia. DBEW Publication (NIH) 79; 1296, Washington: US Government Printing Office, 1979: 167-71.

23 Schwarzenberg SJ and Sharp HL. Pathogenesis of alpha-1antitrypsin deficiency-associated liver disease. $f$ Pediat Gastroenterol Nutr 1990; 10: 5-12.

24 Carrell RW. Alpha-1-antitrypsin: molecular pathology, leukocytes, and tissue damage. $\mathcal{F}$ Clin Invest 1986; 78: 1427-31.

25 Doherty DG, Donaldson PT, Whitehouse DB, et al. HLA phenotypes and gene polymorphisms in juvenile liver disease associated with alpha-1-antitrypsin deficiency. Hepatology 1990; 12: 218-23.

26 Nebbia G, Hadchouel M, Alagille D. Early assessment of evolution of liver disease associated with alpha-1-antitrypsin deficiency in childhood. Pediatrics 1983; 102: 661-8.

27 Psacharopoulos HT, Mowat AP, Cooke PJL, Carlile PA, Portmann B, Rodeck CH. Outcome of liver disease associated with alpha-1-antitrypsin deficiency. Implications for genetic counselling and antenatal diagnosis. Arch Dis Child 1983; 58: 882-7.

28 Noble-Jamieson G, Barnes N, Thiru S, Mowat AP. Severe hypertension after liver transplantation in children with alpha-1-antitrypsin deficiency. Arch Dis Child 1990; 65: 1217-21.

29 Cohen A, O'Grady J, Mowat AP, Williams R. Liver transplantation for metabolic disorders. In: Bailliere's clinical gastroenterology 1989; 3: 767-86.

30 Starzl TE, Demetris J, Van Thiel D. Medical progress: liver transplantation. N Englf Med 1989; 321: 1014-22; 1092-9.

31 Corney G, Whitehouse DB, Hopkinson DA, et al. Prenatal diagnosis of alpha-1-antitrypsin deficiency by fetal blood sampling. Prenatal Diagn 1987; 7: 101-8.

32 Povey $S$ The genetics of alpha-1-antitrypsin deficiency in relation to neonatal liver disease. Molec Biol Med 1990; 7 161-72.

33 Crystal RG. Alpha,-antitrypsin deficiency, emphysema and liver disease. Genetic basis and strategies for therapy. $\mathrm{f}$ Clin Invest 1990; 85: 1343-51.

34 Wu WJ, Wilson JM, Wu Ch. Targeting genes: detection of targeted human albumin gene expression in genetically analbuminaemic rats. Hepatology 1989; 10: 618 .

35 Brandtly M, Courtney M, Crystal RG. Repair of the secretion defect in the $\mathrm{Z}$ form of alpha-1-antitrypsin by the addition of a second mutation. Science 1988; 242: 1700-2.

36 Spence MW, Callahan JW Sphingomyelin-cholesterol Lipidosis; The Niemann-Pick Group of disorders. In: Scriver CR, Beautt AL, Sly WS, Valle D, eds. The metabolic basis of inherited disease 6th ed. New York: McGraw-Hill, 1989: 1479-1509.

37 Vanier MT, Wenger DA, Cowley ME, Rousson R, Brady RO, Pentchev PG. Niemann-Pick disease type C: clinical variability and diagnosis based on defective cholesterol esterification. A collaborative study based on seventy patients. Clin Genet 1988; 33: 331-8.

38 Maconochie IK, Chong S, Mieli-Vergani G, Lake BD, Mowat AP. Fetal ascites: an usual presentation of Niemann-Pick disease type C. Arch Dis Child 1989; 10: 1391-3.

39 Wanders RJA, van Roermund CWT, Schutgens RBH, et al. 
The inborn errors of peroxisomal beta-oxidation: a review. F Inher Metab Dis 1990; 13: 411-8.

40 Lazarow PB, Moser HW. Disorders of Peroxisome biogenesis. In: Scriver CR, Beautt AL, Sly WS, Valle D, eds. The metabolic basis of inherited disease. 6th ed. New York: metabolic basis of inherited disease. 6th ed. New York: 41 Mraw-Hill, 1989: 1479-1509.

of of interlobular bile ducts (Alagille's syndrome or arteriohepatic dysplasia): review of eighty cases. F Pediatr 1987; 110: $195-200$.
42 Deprettere A, Portmann B, Mowat AP. Syndromic paucity of the intra hepatic bile ducts: diagnostic difficulty; severe morbidity throughout early childhood. I Pediatr Gastroenterol Nutr 1987; 6: 865-71.

43 Wilkinson ML, Mieli-Vergani G, Ball C, Portmann B, Mowat AP. Endoscopic retrograde cholangiopancreatography AP. Endoscopic retrograde cholangiopancreatography
(ERCP) in infantile cholestasis. Arch Dis Child 1991; 66: 121-3. 